\title{
Aggregation-prone GFAP mutation in Alexander disease validated using a zebrafish model
}

\author{
So-Hyun Lee ${ }^{1,2+}$, Tai-Seung Nam ${ }^{3+}$, Kun-Hee Kim ${ }^{4}$, Jin Hee Kim ${ }^{4}$, Woong Yoon ${ }^{5}$, Suk-Hee Heo ${ }^{5}$, Min Jung Kim ${ }^{6}$,
} Boo Ahn Shin ${ }^{4}$, Ming-Der Perng ${ }^{7}$, Hyon E. Choy ${ }^{4}$, Jihoon Jo ${ }^{1 *}$, Myeong-Kyu Kim ${ }^{3 *}$ and Seok-Yong Choi ${ }^{1,2^{*}}$ (I)

\begin{abstract}
Background: Alexander disease $(\mathrm{AxD})$ is an astrogliopathy that predominantly affects the white matter of the central nervous system (CNS), and is caused by a mutation in the gene encoding the glial fibrillary acidic protein (GFAP), an intermediate filament primarily expressed in astrocytes and ependymal cells. The main pathologic feature of AxD is the presence of Rosenthal fibers (RFs), homogeneous eosinophilic inclusions found in astrocytes. Because of difficulties in procuring patient' CNS tissues and the presence of RFs in other pathologic conditions, there is a need to develop an in vivo assay that can determine whether a mutation in the GFAP results in aggregation and is thus disease-causing.

Methods: We found a GFAP mutation (c.382G > A, p.Asp128Asn) in a 68-year-old man with slowly progressive gait disturbance with tendency to fall. The patient was tentatively diagnosed with AxD based on clinical and radiological findings. To develop a vertebrate model to assess the aggregation tendency of GFAP, we expressed several previously reported mutant GFAPs and p.Asp128Asn GFAP in zebrafish embryos.

Results: The most common GFAP mutations in AxD, p.Arg79Cys, p.Arg79His, p.Arg239Cys and p.Arg239His, and p. Asp128Asn induced a significantly higher number of GFAP aggregates in zebrafish embryos than wild-type GFAP.

Conclusions: The p.Asp128Asn GFAP mutation is likely to be a disease-causing mutation. Although it needs to be tested more extensively in larger case series, the zebrafish assay system presented here would help clinicians determine whether GFAP mutations identified in putative AxD patients are disease-causing.
\end{abstract}

Keywords: Alexander disease, Leukodystrophy, Glial fibrillary acidic protein, Rosenthal fibers, Astrocyte, Zebrafish, GFAP

\section{Background}

Alexander disease $(\mathrm{AxD})$ is a neurodegenerative disorder that primarily affects the white matter of the central nervous system (CNS) [1-5]. It was first reported in 1949 by W. Stewart Alexander in a 15-month-old boy with megalencephaly, hydrocephalus and psychomotor retardation. The brain pathology of the boy showed "progressive fibrinoid degeneration of fibrillary astro-

\footnotetext{
*Correspondence: Jihoon.Jo@chonnam.ac.kr; mkkim@chonnam.ac.kr; zebrafish@chonnam.ac.kr

${ }^{\dagger}$ Equal contributors

${ }^{1}$ Department of Biomedical Sciences, Chonnam National University Medical School, Gwangju 501-759, Republic of Korea

${ }^{3}$ Department of Neurology, Chonnam National University Medical School, Gwangju 501-759, Republic of Korea

Full list of author information is available at the end of the article
}

cytes," [6] which was later identified as Rosenthal fibers that were initially described by Werner Rosenthal in ependymoma in 1898 [7]. Rosenthal fibers are homogeneous eosinophilic inclusions stained by hematoxylin and eosin, and consist mainly of glial fibrillary acidic protein (GFAP), $\alpha B$-crystallin, heat shock protein (HSP) 27 and cyclin D2 [2, 3, 5]. Messing and colleagues reported that $\mathrm{AxD}$ was elicited by mutations in the gene encoding GFAP, a type III intermediate filament predominantly found in astrocytes. They suggested that the mutations act in a gain-of-function fashion based on their finding that the phenotypes of Gfap null mice did not parallel those of AxD [8]. Since then, many different GFAP mutations have been reported in $\mathrm{AxD}$ patients [9]. 
AxD has been classified into three clinical subtypes depending on age at onset (AAO). Infantile AxD (birth to 2 years), the most frequent subtype, is characterized by progressive megalencephaly and/or hydrocephalus, developmental delay, psychomotor retardation, epileptic seizures. Juvenile $\operatorname{AxD}$ (2-14 years) features spastic paraplegia, progressive bulbar signs and ataxia with spared cognitive function. Adult AxD (late adolescence and beyond), the least frequent subtype and often misdiagnosed with multiple sclerosis, shows variable manifestations including progressive ataxia, tetraparesis, bulbar and pseudobulbar signs [3, 10]. A revised classification system was proposed based on statistical analysis of clinical, radiologic, and genetic features of 215 cases of AxD. In the revised system, patients with type I AxD show early AAO, macrocephaly, developmental delay and typical brain magnetic resonance imaging (MRI) features. In contrast, patients with type II AxD exhibit various AAO, bulbar symptoms, ocular movement abnormality and atypical MRI findings [11].

Although AxD can be diagnosed based on comprehensive evaluation of patient history, physical examination, brain MRI, GFAP sequencing and cerebral biopsy, GFAP sequencing and cerebral biopsy remain to be the best diagnostic approaches [3, 10]. Detection of Rosenthal fibers through cerebral biopsy is considered to be one of the best diagnostic approaches. However, most putative AxD patients with GFAP mutations did not undergo cerebral biopsy [12-14] as it is an invasive procedure. In addition, Rosenthal fibers are not a pathognomonic feature of $\mathrm{AxD}$ because they are also occasionally found in astrocytic tumors, ependymoma, hamartomas, craniopharyngioma, pineal cysts, glial scars and multiple sclerosis $[3,15]$. Hence, DNA sequencing is the only definitive diagnostic approach for AxD under most circumstances. However, identification of GFAP mutations in putative AxD patients does not guarantee that these mutations are associated with AxD because it is feasible that these mutations are just variants of unknown significance. Therefore, it is imperative to determine whether the GFAP mutations found in tentative AxD patients are disease-causing. To this end, two methods have been employed. First, an in vitro assembly assay was performed with recombinant mutant GFAPs purified from $E$. coli and the formation of aggregates was then assessed. Second, an expression plasmid encoding the mutant GFAP was transfected into various mammalian cell lines, which were then observed for GFAP aggregates [13, 16-20]. However, these methods might not be suitable for testing the causality of the GFAP mutations, because both methods do not reflect the in vivo environment around astrocytes and the second method adopts a strong exogenous promoter to express mutant GFAP.

Zebrafish (Danio rerio) are tropical freshwater fish and a vertebrate model organism that is used to study vertebrate development because of transparent embryos, and rapid and external development. Especially, zebrafish have been extensively used to research nervous system development and to establish vertebrate models of neurodegenerative diseases [21, 22]. Zebrafish have astrocytes [23], and zebrafish Gfap shares $67 \%$ identity and $77 \%$ similarity with human GFAP, along with well-conserved hot spot amino acids mutated in AxD (Fig. 1a) [24]. In addition, regulatory elements that drive the specific expression of zebrafish gfap in astrocytes were identified [25].

We saw a patient who presented with slowly progressive gait disturbance and a missense mutation in the GFAP, and made a tentative diagnosis of $\mathrm{AxD}$ based on clinical and radiological findings. To determine whether the mutation is disease-causing, we set out to develop a zebrafish model that would be useful for molecular diagnosis of $\mathrm{AxD}$.

\section{Methods \\ Reagents}

All chemicals were purchased from Sigma (St. Louis, $\mathrm{MO})$, unless indicated otherwise.

\section{DNA sequencing}

Genomic DNA (gDNA) was extracted from the peripheral blood of subjects using a Wizard Genomic DNA purification kit (Promega, Madison, WI), and all nine exons and exon-intron boundaries of the GFAP were PCR-amplified from the extracted gDNA as described previously [20, 26].

\section{DNA manipulation}

For the expression study, human GFAP was PCR-amplified from GFAP cDNA (NCBI accession number BC013596, Dharmacon, Lafayette, $\mathrm{CO}$ ) with specific primers (Table 1), and the resulting PCR product was cloned into the BamHI/ EcoRV sites of the pCS4 + -3xFLAG-P2A vector [27]. p.Arg79Cys, p.Arg79His, p.Arg239Cys, p.Arg239His and p.Asp128Asn mutations were individually inserted into the WT GFAP construct by site-directed mutagenesis with specific primers (Table 1). For zebrafish study, the zebrafish gfap regulatory elements $(7.4 \mathrm{~kb})$ [25] were cloned into the BglII/SalI sites of a mini-Tol2 (T2AL200R150G) plasmid [28]. EGFP and human GFAP C-terminally fused to a FLAG epitope were then sequentially cloned into the resulting construct (Fig. 2b). All plasmids constructed were verified by DNA sequencing (Macrogen, Daejeon, Korea).

\section{Cell culture and western blotting}

HEK293T cells were purchased from American Type Culture Collection (Manassas,VA), cultured in Dulbecco's modified Eagle's media (Welgene, Daegu, Korea) supplemented with $10 \%$ fetal bovine serum (Thermo 
A
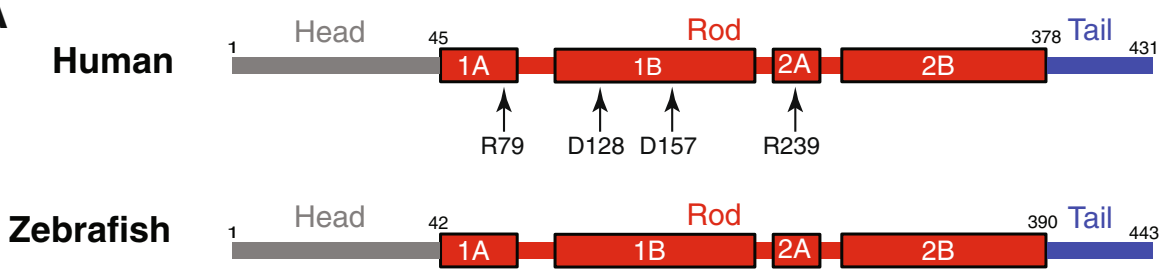

B
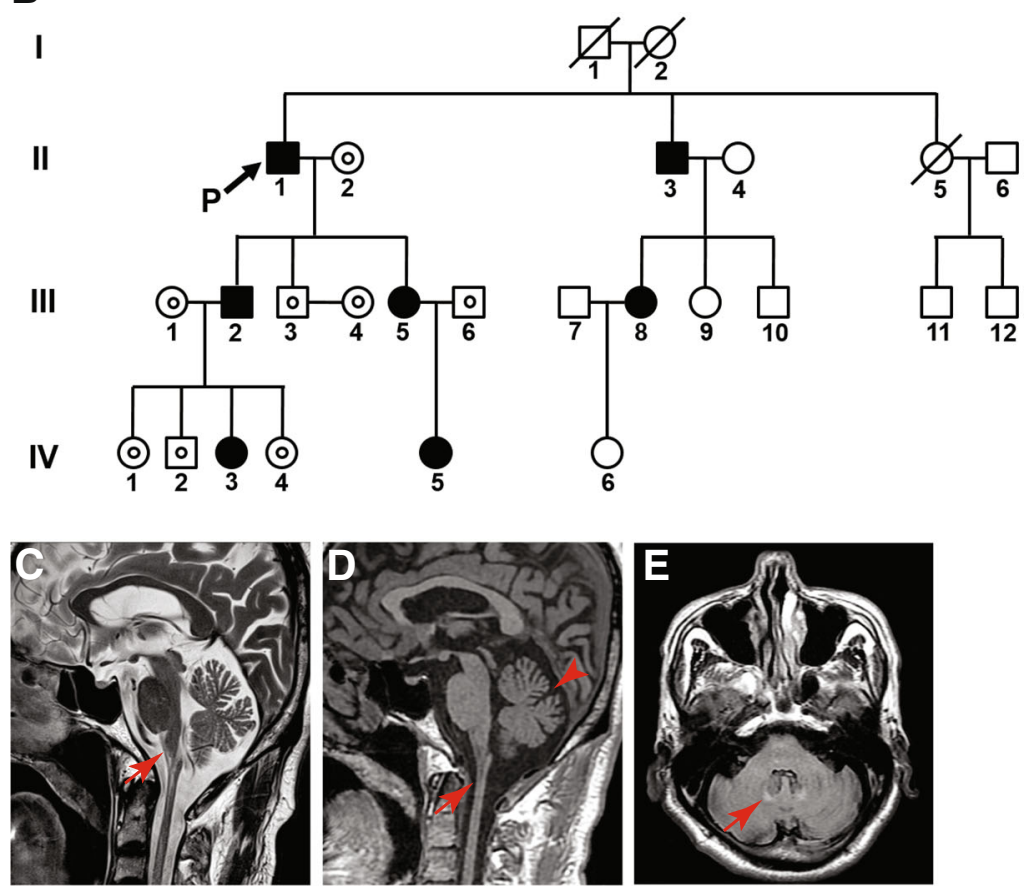

$\mathbf{F}$

G

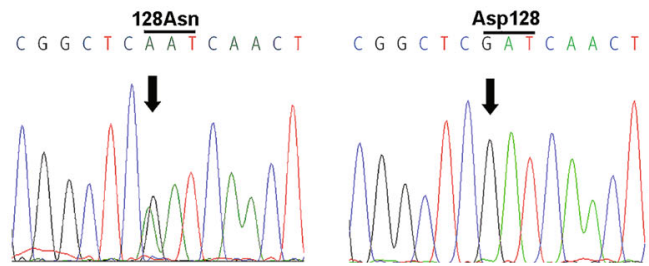

Fig. 1 Clinical features and GFAP sequences of the proband. a Comparison between human and zebrafish GFAP, and location of amino acid residues whose mutations are discussed in this study. Human GFAP: NCBI accession number NP_002046; zebrafish Gfap: NP_571448. D: aspartate; R: arginine. b Pedigree of individuals with p.Asp128Asn GFAP shown as solid symbols. Symbols and nomenclature follow established guidelines [44]. A small circle within a square or a circle indicates an individual who tested negative for a GFAP mutation. P, proband. c-e Brain MR images of the proband. c Sagittal T2-weighted MR image shows marked atrophy of the medullar oblongata (arrow). d Sagittal T1-weighted MR image reveals prominent atrophy in the upper cervical cord (arrow) and cerebellar hemisphere (arrowhead). e Fluid-attenuated inversion recovery (FLAIR) image shows high signal intensity lesions in the bilateral cerebellar dentate nuclei (arrow). $\mathbf{f}$ and $\mathbf{g}$ DNA sequence analysis of the GFAP. Arrows indicate c.382G. f Electropherogram of the proband reveals a heterozygous G-to-A substitution at position 382 of the GFAP, which is predicted to substitute asparagine for aspartic acid (p.Asp128Asn). g Representative electropherogram of GFAP sequences in 200 control subjects

Fisher Scientific Korea, Seoul, Korea), and transfected with plasmid using Lipofectamine 2000 (Thermo Fisher Scientific Korea) according to the manufacturer's instructions. Subsequently, the cells were lysed with MPER mammalian protein extraction reagent (Thermo Fisher Scientific Korea) at $48 \mathrm{~h}$ post-transfection, and processed for Western blotting as described previously
[27]. The antibodies used were anti-FLAG antibody (1:2000, Sigma-Aldrich, catalog number F1804), anti-glyceraldehyde-3-phosphate dehydrogenase (anti-GAPDH) antibody (1:2000, Trevigen, Gaithersburg, MD, 2275PC-100), HRP-conjugated goat anti-mouse antibody (1:4000, Santa Cruz Biotechnology, Dallas, TX, sc2005), and HRP-conjugated goat anti-rabbit antibody 
Table 1 Sequences of primers $\left(5^{\prime} \rightarrow 3^{\prime}\right)$ used to construct plasmids encoding various human GFAP alleles

\begin{tabular}{ll}
\hline Allele & Sequences \\
\hline WT & Forward: TAGTAGGATCCATGGAGAGGAGACGCATCAC \\
& Reverse: TAGTCGATATCATCATCACATCCTTGTGCTCC \\
& TGCTTG \\
p.Arg79Cys & Forward: GAGATGATGGAGCTCAATGACtGCTTTGCCA \\
& GCTACATCGAG
\end{tabular}

Reverse: CTCGATGTAGCTGGCAAAGCaGTCATTGAGC TCCATCATCTC

p.Arg79His Forward: GAGATGATGGAGCTCAATGACCaCTTTGCC
AGCTACATCGAG

Reverse: CTCGATGTAGCTGGCAAAGtGGTCATTGAGC TCCATCATCTC

p.Asp128Asn Forward: GAGAGCTGCGGCTGCGGCTCaATCAACTCA CCGCCAACAG

Reverse: CTGTTGGCGGTGAGTTGATtGAGCCGCAGCC GCAGCTCTC

p.Asp157Asn Forward: GCAGAAGCTCCAGaATGAAACCAACCTG

Reverse: CAGGTTGGTTTCATtCTGGAGCTTCTGC

p.Arg239Cys Forward: CAGCCCTGAAAGAGATCtGCACGCAGTAT GAGGCAATG

Reverse: CATTGCCTCATACTGCGTGCaGATCTCTTT CAGGGCTG

p.Arg239His Forward: CAGCCCTGAAAGAGATCCaCACGCAGTATGA GGCAATG

Reverse: CATTGCCTCATACTGCGTGtGGATCTCTTTCA GGGCTG

Lower case indicates mutated nucleotides

(1:4000, Santa Cruz Biotechnology, sc-2004). Band intensity on the Western blots was analyzed using ImageJ.

\section{Zebrafish study}

Wild-type (WT) zebrafish (AB strain) were obtained from the Zebrafish International Resource Center (Eugene, OR), maintained using standard procedures [29] and staged in hours post-fertilization (hpf) as per standard criteria [30]. One-cell stage zebrafish embryos were microinjected with GFAP expression constructs
(50 pg), anesthetized at $30 \mathrm{hpf}$ in $0.02 \%$ tricane, mounted with 3\% methylcellulose and imaged with an LSM 510 CLM (Zeiss, Hamburg, Germany). Z-series of images (15 images; interval thickness: $1.0 \mu \mathrm{m}$ ) were collected and presented as a stacking image. Resulting images were assembled using Adobe Photoshop (San Jose, CA), and aggregations were counted blindly.

\section{Statistical analysis}

$P$ values [31] were determined with the two-tailed paired Student's t test. $P<0.05$ was considered statistically significant.

\section{Transmission electron microscopy (TEM)}

TEM was performed in the Electron Microscopy Facility at Yonsei Biomedical Reseach Institute at Yonsei University College of Medicine. In brief, zebrafish embryos injected with expression plasmids encoding WT or p.Arg79Cys GFAP were fixed at $30 \mathrm{hpf}$ in $0.1 \mathrm{M}$ phosphate buffer ( $\mathrm{pH}$ 7.4) with $2 \%$ glutaraldehyde (Merck, Darmstadt, Germany) and paraformaldehyde (Merck) for $12 \mathrm{~h}$, washed in $0.1 \mathrm{M}$ phosphate buffer, post-fixed with $1 \% \mathrm{OsO}_{4}$ in $0.1 \mathrm{M}$ phosphate buffer for 90 min, dehydrated with an ascending ethanol series (50\%, 60\%, 70\%, 80\%, 90\%, 95\% and 100\%) for $10 \mathrm{~min}$ each, and infiltrated with propylene oxide for $10 \mathrm{~min}$. Subsequently, specimens were embedded with a Poly/ Bed 812 embedding kit (Polysciences, Warrington, PA), polymerized in an electron microscope oven (TD-700, DOSAKA, Kyoto, Japan) at $65{ }^{\circ} \mathrm{C}$ for $12 \mathrm{~h}$, cut into $200 \mathrm{~nm}$ thick semi-thin sections using an EM UC7 ultramicrotome (Leica Microsystems, Wetzlar, Germany) with a diamond knife (DiATOME, Hatfield, PA), stained with toluidine blue and observed with a light microscope. The region of interest was then cut into $80 \mathrm{~nm}$ thick ultra-thin sections using the ultramicrotome, placed on copper grids, stained in 4\% uranyl acetate (Electron Microscopy Sciences, Hatfield, PA) for $20 \mathrm{~min}$ followed by lead citrate (Thermo Fisher Scientific Korea) for $10 \mathrm{~min}$, and imaged with a transmission electron

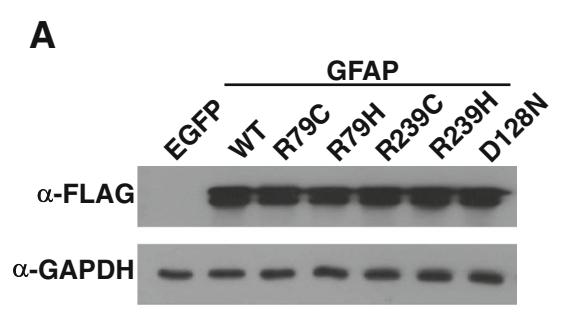

B

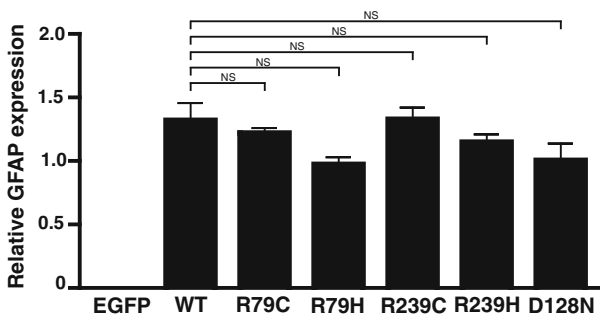

Fig. 2 Protein expression levels of mutant alleles were comparable to that of WT GFAP. a HEK293T cells were transfected with plasmid encoding EGFP or indicated alleles of GFAP C-terminally fused to a FLAG epitope, and processed for Western blotting with anti-FLAG antibody. Anti-GAPDH (glyceraldehyde-3-phosphate dehydrogenase) antibody was used as a loading control. b Quantitation of GFAP band intensity in (a) normalized to GAPDH band intensity $(n=3)$. NS: not significant 
microscope (JEM-1011, JEOL, Tokyo, Japan) equipped with a MegaView III CCD camera (Olympus Soft Imaging Solutions, Lakewood, $\mathrm{CO}$ ) at the acceleration voltage of $80 \mathrm{kV}$.

\section{Results}

\section{A 68-year-old male with ataxia}

A 68-year-old Korean man (proband; subject II.1 in Fig. 1b), who exhibited slowly progressive gait disturbance with tendency to fall for several months, was referred to our hospital. Albeit self-ambulatory, the proband suffered from unsteady gait due to ataxia. His medical history was unremarkable except for mild hypertension. The proband had no family history of neurological diseases or consanguineous marriage. Neurological examination revealed dysphagia, dysarthria, dysphonia, wide-based truncal ataxia, bilateral gaze-evoked nystagmus and exaggerated deep tendon reflexes with bilaterally positive Babinski and Hoffman signs, indicating dysfunctions in the brainstem, cerebellum or cervical cord. The proband did not present with sensory disturbance, palatal myoclonus, and abnormal mental or emotional status. To determine the etiology of ataxia, extensive workup was carried out including somatosensory evoked potential recording, blood and urine chemistry tests, assay of serum levels of vitamin B12 and thyroid hormones, venereal disease research laboratory (VDRL) test, anti-human immunodeficiency virus (HIV) antibody test and genetic studies for spinocerebellar ataxia (types 1, 2, 3, 6, 7 and 8), Friedreich ataxia and dentato-rubropallido-luysian atrophy. All of these tests were negative, however.

The brain MR images revealed marked atrophy of the medulla oblongata and upper cervical cord, and mild atrophy of the cerebellar hemisphere on both sagittal T2and T1-weighted images (Fig. 1c and d, respectively). Moreover, fluid-attenuated inversion recovery (FLAIR) imaging illustrated hyperintense lesions in the bilateral cerebellar dentate nuclei (Fig. 1e). These MRI findings suggested adult $\mathrm{AxD}$. Therefore, we had the proband's GFAP sequenced and found a heterozygous mutation, c.382G > A, which was absent in the GFAP sequences from 200 normal controls. This substitution was predicted to lead to p.Asp128Asn (Fig. If and g). Sequencing of the GFAP of the proband's family members suggested Mendelian inheritance of the mutation (Fig. 1b). Out of the other family members with p.Asp128Asn, subjects II.3, III.2, and III.5 showed hyperreflexia of the upper and lower extremities with positive Babinski and Hoffman signs and without evidence of neurological symptoms (Fig. 1b), indicating early stage of adult onset $\mathrm{AxD}$ or various degrees of penetrance.

p.Asp128Asn was reported in two cases of $\mathrm{AxD}$, and Rosenthal fibers in the brain were observed posthumously in one of the cases [32,33]. Albeit characteristic,
Rosenthal fibers are not pathognomonic of $\operatorname{AxD}[3,15]$. As such, we turned to a zebrafish model to test if p.Asp128Asn GFAP is disease-causing.

\section{Zebrafish can be used to assess the formation of mutant GFAP aggregates}

Of mutant GFAPs reported in AxD patients, the most common are p.Arg79Cys, p.Arg79His, p.Arg239Cys and p.Arg239His [9]. To test if these mutant GFAPs aggregate in zebrafish embryos, we first generated expression plasmids individually encoding WT or one of the four GFAP mutants C-terminally fused to a FLAG epitope, and compared their expression levels in human embryonic kidney HEK293T cells by Western blotting. Expression levels of all of the mutants were comparable to that of WT GFAP (Fig. 2a and b), indicating that the four mutation alleles do not affect GFAP stability. We next individually cloned the WT or mutant alleles of GFAP C-terminally fused to a FLAG epitope and enhance green fluorescent protein (EGFP) into the 3' end of the zebrafish gfap promoter [25], and the resulting constructs (Fig. 3a) were microinjected into one-cell stage zebrafish embryos. Subsequently, brain and trunk regions of the embryos expressing comparable levels of GFP at $30 \mathrm{hpf}$ were imaged with a confocal laser microscope (CLM; Fig. 3b). Embryos microinjected with WT GFAP plasmids showed GFP aggregations. This was not surprising as supplementation of human GFAP to zebrafish that have their own GFAP proteins expressed, could lead to GFAP aggregation zebrafish. This is supported by the previous report that expression of WT human GFAP in mouse triggered aggregation of GFAP [34]. Nevertheless, the number of aggregations was significantly higher in both the head and trunk regions of the embryos microinjected with plasmids encoding common GFAP mutants (Fig. 3c, d, and e). To further validate this method as a tool to determine pathogenicity of GFAP mutations, we repeated the experiment with p.Asp157Asn GFAP that was previously reported to be a non-disease causing variant [13]. As expected, so significant difference in aggregation was noted between WT and p.Asp157Asn GFAP (Fig. 3f-h).

To check if GFP aggregations in zebrafish embryos are akin to GFAP aggregations in $\mathrm{AxD}$ patients, we performed transmission electron microscopy (TEM) on zebrafish embryos microinjected with expression plasmids encoding WT or p.Arg79Cys allele of GFAP, and indeed found electron dense inclusions in the cells of both groups of embryos (Fig. 4a-c), which is reminiscent of TEM findings of RFs in the astrocytes of the AxD brain [35]. Of note, more inclusions were observed in the TEM images of p.Arg79Cys embryos than WT embryos, consistent with CLM images. Intriguingly, found in the p.Arg79Cys embryos were the spherical structures 


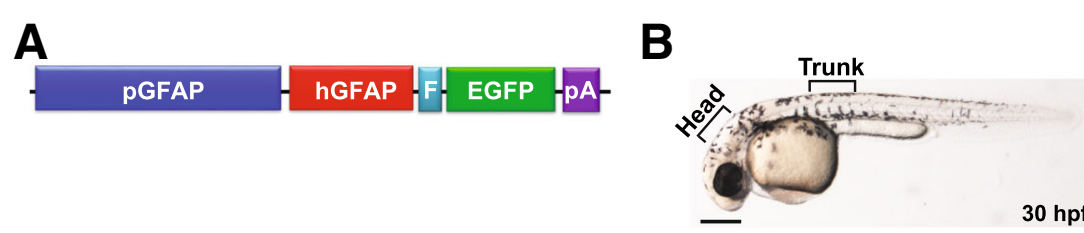

C

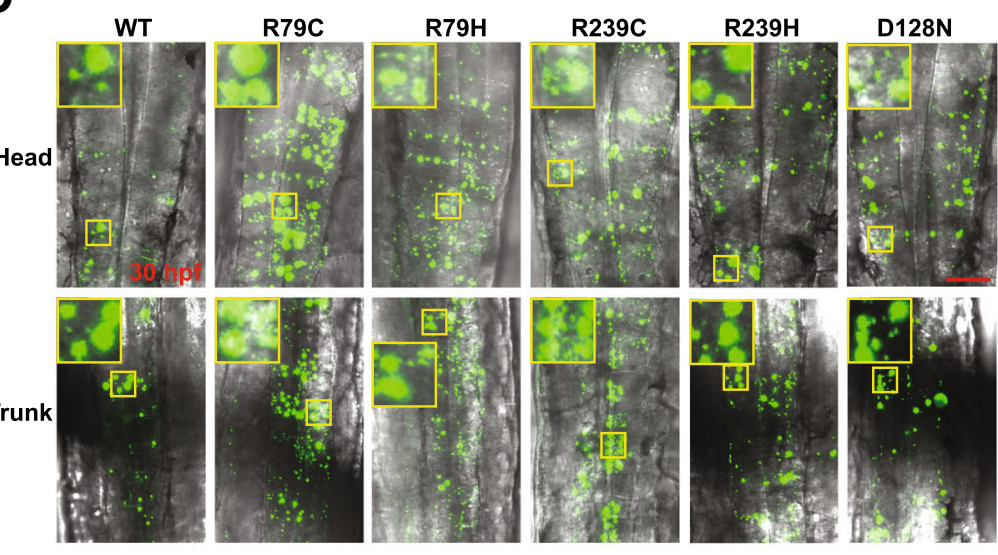

D

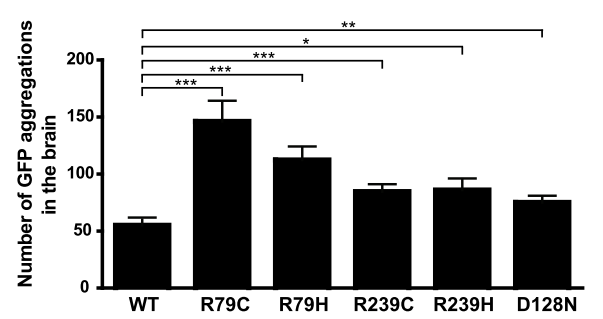

$\mathbf{F}$

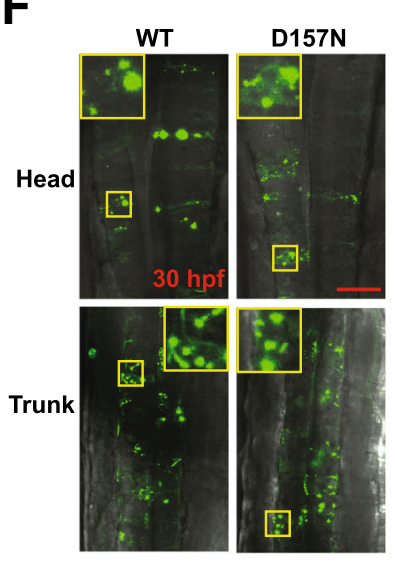

E

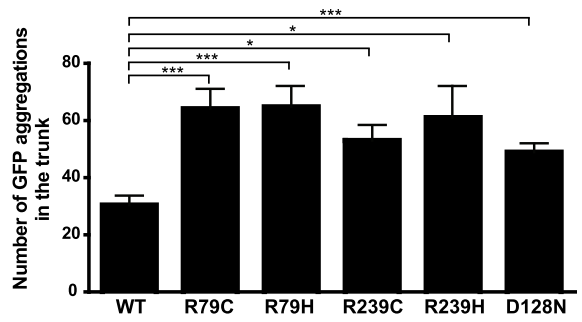

H

G
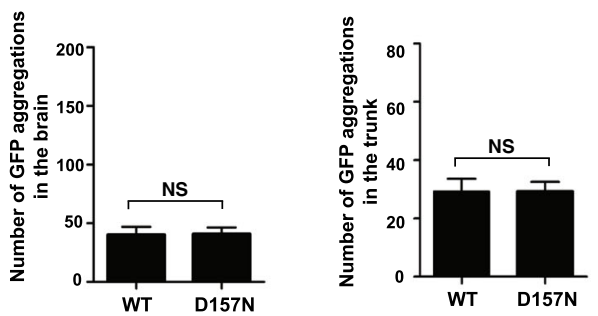

Fig. 3 Aggregation susceptibility of mutant GFAPs can be assessed using zebrafish. a Schematic representation of an expression plasmid encoding human GFAP C-terminally fused to a FLAG epitope and EGFP driven by a zebrafish gfap promoter. EGFP: enhanced green fluorescent protein; F: 3x FLAG epitope tag; hGFAP: human GFAP; pA: polyadenylation sequence; and pGFAP: zebrafish gfap promoter. b Regions of zebrafish embryos at $30 \mathrm{~h}$ post-fertilization (hpf) imaged in (c). c One-cell stage zebrafish embryos were microinjected with expression plasmids encoding WT or indicated alleles of GFAP and imaged with a confocal laser microscope at $30 \mathrm{hpf}$. Images represent stacking of Z-series of images. Insets represent magnifications of the boxed areas. R79C: p.Arg79Cys; R79H: p.Arg79His; R239C: p.Arg239Cys; R239H: p.Arg239His; and D128N:

p.Asp128Asn. Scale bar $=150 \mu \mathrm{m}$. $\mathbf{d}$ and $\mathbf{e}$ GFP aggregates, indicated by green dots, were counted in the brain (d) and trunk (e) regions of each group in (c). $\mathrm{n}=\mathrm{WT}$ : 10; R79C: 9; R79H: 12; R239C: 15; R239H: 8; and D128N: 11. *: $P<0.05$; *: $P<0.01$; ***: $P<0.001$. f Aggregation assays were performed with WT or D157N allele of GFAP as described in (c). Insets represent magnifications of the boxed areas. D157N: p. Asp157Asn. $\mathbf{g}$ and $\mathbf{h}$ GFP aggregates were counted as described in (d and $\mathbf{e}$ ). NS, not significant. Scale bar $=150 \mu \mathrm{m}$ 

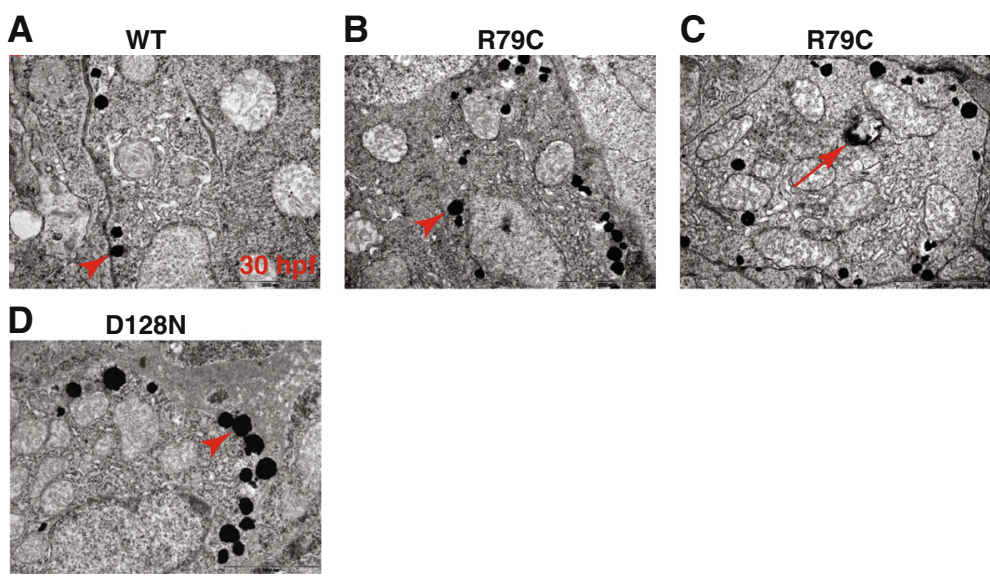

Fig. 4 Aggregation susceptibility of mutant GFAPs can be assessed using zebrafish. a-d Zebrafish embryos at one-cell stage were microinjected with expression plasmids encoding WT (a), p.Arg79Cys GFAP (b and c), or p.Asp128Asn (d), and imaged at 30 hpf with transmission electron microscopy. Arrows and arrowhead indicate electron dense inclusions and a spherical structure with double-layered membranes, respectively. Scale bar $=2 \mu \mathrm{m}$

with double layer membranes containing electron dense inclusions (Fig. 4c). These were reminiscent of autophagosome, which were previously reported in the AxD patient's brain, mouse brain expressing p.Arg236His, and human astrocytoma U251 cells expressing p.Arg239Cys $[35,36]$. Taken together, this outcome indicates that the GFAP aggregation assay in zebrafish embryos can be employed to assess the pathogenicity of GFAP mutations identified in patients tentatively diagnosed with AxD.

\section{P.Asp128Asn induces a significantly higher number of GFAP aggregations in the zebrafish embryos compared to WT GFAP}

To ascertain whether the p.Asp128Asn GFAP allele of the proband is aggregation prone, we first compared expression levels between p.Asp128Asn GFAP and WT GFAP in HEK293T cells by Western blotting and observed comparable expression levels of the two GFAP alleles (Fig. 2a and b), demonstrating that the p.Asp128Asn does not affect GFAP stability. We next repeated the aggregation assay in zebrafish embryos with p.Asp128Asn GFAP plasmids, and found that p.Asp128Asn induced a significantly higher number of GFP aggregations in both head and trunk regions of the embryos compared to GFAP WT (Fig. 3c-e). Moreover, TEM revealed more electron dense inclusions in p.Asp128Asn embryos than WT embryos (Fig. 4a, d). These findings indicate that p.Asp128Asn is aggregation prone and thus may cause AxD.

\section{Discussion}

Here, we show that a 68-year-old male with ataxia and atrophy of the medulla oblongata, upper cervical cord and the cerebellar hemispheres on brain MRI harbors a p.Asp128Asn GFAP mutation. Furthermore, we demonstrate that the p.Asp128Asn mutation induces more GFAP aggregations in zebrafish embryos than WT GFAP, suggesting that this mutation may cause AxD.

Most neurodegenerative diseases are protein-misfolding disorders (PMDs), and animal models of PMDs are instrumental in addressing many important questions about their molecular pathogeneses and the development of therapeutic modalities. Hence, several model organisms have been utilized to generate animal models of PMDs. For example, Hart and colleagues expressed polyglutamine tracts in the ASH sensory neurons of Caenorhabditis elegans to model Huntington's disease and found neurodegeneration and apoptosis of ASH neurons [37]. Zhong and colleagues expressed amyloid- $\beta$ peptides $A \beta 42$ in the neurons of Drosophila melanogaster and noted amyloid deposits, late-onset progressive neurodegeneration and olfactory learning defects [38]. Ayyagari and colleagues showed that a mutant allele of asparaginase like-1 (ASRGL1) identified in a family with inherited retinal degeneration induced protein aggregation in monkey kidney fibroblast-like COS-7 cells, and retinal photoreceptor degeneration in zebrafish larvae [39]. Hsiao and colleagues generated Tg2576 transgenic mice expressing a Swedish allele of amyloid precursor protein and observed impaired learning and memory and the deposition of amyloid plaques in the brain [19]. Although invertebrate model organisms such as $C$. elegans and D. melanogaster have been used to model PMDs, they have certain limitations. First, they lack key factors critical to many human PMD pathogeneses, such as myelination, specialized neuronal and glial cell types, and a sophisticated immune system. Second, the anatomical structures of their brains are quite divergent from those of humans [40]. On the other hand, zebrafish are vertebrates such that zebrafish can overcome 
aforementioned limitations. In addition, zebrafish present other advantages as a model of PMDs, such as small size, transparency, and external embryonic development. Thus, zebrafish can be used not only to investigate the molecular pathogenesis of PMDs, but also to develop therapeutics against PMDs. For example, inhibitors of polyglutamine protein aggregation were identified using zebrafish embryos [41]. As such, the zebrafish GFAP aggregation model we present here will help elucidate the molecular pathogenesis of $\mathrm{AxD}$ and serve as a basis for the development of AxD therapeutics.

In the present study, plasmids encoding mutant GFAPs were injected into zebrafish embryos at $1 \mathrm{hpf}$, and GFP aggregates in the embryos were imaged at $30 \mathrm{hpf}$ (Fig. 2d). This signifies that the zebrafish embryo assay system can determine aggregation tendency of mutant GFAPs in less than two days. Therefore, this system would be useful for clinicians to make a swift and accurate diagnosis of AxD.

We demonstrated in zebrafish embryos that the p.Asp128Asn induced less GFAP aggregates than p.Arg79Cys, p.Arg79His, p.Arg239Cys and p.Arg239His. The proband's AxD appears to be classified as adult or type II AxD given the proband's late AAO, bulbar symptoms, nystagmus and atypical MRI features $[3,10,11]$. Two previously reported cases of p.Asp128Asn also seem to be of the same classification [32, 33]. On the other hand, almost all cases of p.Arg79Cys, p.Arg79His, p.Arg239Cys and p.Arg239His fall under the infantile or type I AxD classification [11]. It is therefore tempting to speculate that the aggregation tendency of GFAP mutants may be related to AAO: high aggregation tendency results in early AAO, thereby infantile or type I AxD, and low aggregation tendency brings about late AAO, leading to adult or type II AxD. This notion is supported by a report by Perng and colleagues that two mutant alleles of GFAP found in infantile AxD, p.Asn386Ile and p.Asp417MetfsX14, induced more GFAP aggregates than did three mutant alleles found in adult AxD, p.Ser393Ile, p.Ser398Phe and p.Ser398Tyr [42]. Of course, this notion warrants further comprehensive investigation.

Messing and colleagues reported that p.Arg239His GFAP increased Gfap promoter activity in mice compared to WT GFAP [43]. This finding may be extended to other pathogenic GFAP mutations. If this is the case, higher aggregations we observed for pathogenic GFAP mutations might ensue from higher GFAP expression, at least in part. This notion warrants further investigation.

\section{Conclusions}

Establishment of a zebrafish embryo assay system that could be employed to assess in vivo susceptibility of GFAP to aggregation, would help clinicians determine whether GFAP mutations identified in putative AxD patients are disease-causing.

\author{
Abbreviations \\ AAO: Age at onset; AxD: Alexander disease; CLM: Confocal laser microscope; \\ CNS: Central nervous system; EGFP: Enhance green fluorescent protein; \\ FLAIR: Fluid-attenuated inversion recovery; gDNA: genomic DNA; GFAP: Glial \\ fibrillary acidic protein; HIV: Human immunodeficiency virus; Hpf: Hours post- \\ fertilization; HSP: Heat shock protein; MRI: Magnetic resonance imaging; \\ PMD: Protein-misfolding disorder; RF: Rosenthal fiber; TEM: Transmission \\ electron microscopy; VDRL: Venereal disease research laboratory; WT: Wild-type
}

\section{Acknowledgements}

We thank Nan Hee Choi for technical assistance, Pamela Raymond for zebrafish gfap regulatory elements, Koichi Kawakami for mini-Tol2 (T2AL200R150G) plasmid, Eun Jin Kim for transmission electron microscopy and Eun Young Choi for encouragement.

\section{Funding}

This work was supported in part by grants from the Basic Science Research Program through the National Research Foundation of Korea (NRF) funded by the Ministry of Education (2013-R1A1A1058252), the Chonnam National University Hospital Biomedical Research Institute (CRI 12055-21 and CRI 13902-24), and the Chonnam National University Hwasun Hospital Biomedical Research Institute (HCRI 15013-21). These funding sources had no role in the design of the study and collection, analysis, and interpretation of data.

Availability of data and materials

All data generated or analyzed during this study are available from the corresponding authors on reasonable request.

\section{Authors' contributions}

TSN, MKK and SYC conceived and designed the experiments. SHL, KHK and JHK performed the experiments. WY analysed MR images. TSN, SHL, JHK, WY, SHH, MJK, BAS, MDP, HEC, JJ, MKK and SYC analysed and interpreted the data. TSN, JJ, MKK and SYC wrote the manuscript. All authors read and approved the final manuscript.

\section{Ethics approval and consent to participate}

The study protocol was approved by the Institutional Review Board at Chonnam National University Hospital (CNUH-2014-066), and conducted in accordance with the 1964 Declaration of Helsinki and its later amendments. Written informed consents for the use of the medical records and GFAP gene sequences were obtained from all the participants including members of the proband's family. Zebrafish study was granted a waiver of ethics approval by the Chonnam National University Medical School Institutional Animal Care and Use Committee.

\section{Consent for publication}

Written informed consent was obtained from the patient for publication of this case report.

\section{Competing interests}

The authors declare that they have no competing interests.

\section{Publisher's Note}

Springer Nature remains neutral with regard to jurisdictional claims in published maps and institutional affiliations.

\section{Author details}

${ }^{1}$ Department of Biomedical Sciences, Chonnam National University Medical School, Gwangju 501-759, Republic of Korea. ${ }^{2}$ Center for Creative Biomedical Scientists at Chonnam National University, Gwangju, Republic of Korea. ${ }^{3}$ Department of Neurology, Chonnam National University Medical School, Gwangju 501-759, Republic of Korea. ${ }^{4}$ Department of Microbiology,

Chonnam National University Medical School, Gwangju, Republic of Korea. ${ }^{5}$ Department of Radiology, Chonnam National University Medical School, Gwangju, Republic of Korea. ${ }^{6}$ Department of Biological Sciences, Sookmyung Women's University, Seoul, Republic of Korea. ${ }^{7}$ Institute of Molecular Medicine, College of Life Sciences, National Tsing Hua University, Hsinchu, Taiwan. 


\section{Received: 27 February 2017 Accepted: 3 August 2017} Published online: 07 September 2017

\section{References}

1. Alexander Diesease [http://www.ncbi.nlm.nih.gov/books/NBK1172/].

2. Heaven MR, Flint D, Randall SM, Sosunov AA, Wilson L, Barnes S, Goldman JE, Muddiman DC, Brenner M. Composition of Rosenthal fibers, the protein aggregate Hallmark of Alexander disease. J Proteome Res. 2016;15(7):2265-82.

3. Johnson AB, Brenner M. Alexander's disease: clinical, pathologic, and genetic features. J Child Neurol. 2003;18(9):625-32.

4. Quinlan RA, Brenner M, Goldman JE, Messing A. GFAP and its role in Alexander disease. Exp Cell Res. 2007;313(10):2077-87.

5. Sawaishi Y. Review of Alexander disease: beyond the classical concept of leukodystrophy. Brain and Development. 2009;31(7):493-8.

6. Alexander WSProgressive fibrinoid degeneration of fibrillary astrocytes associated with mental retardation in a hydrocephalic infant. Brain 1949, 72(3):373-381, $373 \mathrm{pl}$.

7. Rosenthal W. Über eine eigenthümliche, mit Syringomyelie complicirte Geschwulst des Rückenmarks. Bietr Pathol Anat. 1898;23:111-43.

8. Brenner $M$, Johnson AB, Boespflug-Tanguy $O$, Rodriguez D, Goldman JE, Messing A. Mutations in GFAP, encoding glial fibrillary acidic protein, are associated with Alexander disease. Nat Genet. 2001;27(1):117-20.

9. Alexander disease [http://www.waisman.wisc.edu/alexander-disease/].

10. Mignot C, Boespflug-Tanguy O, Gelot A, Dautigny A, Pham-Dinh D, Rodriguez D. Alexander disease: putative mechanisms of an astrocytic encephalopathy. Cell Mol Life Sci. 2004;61(3):369-85.

11. Prust M, Wang J, Morizono H, Messing A, Brenner M, Gordon E, Hartka T, Sokohl A, Schiffmann R, Gordish-Dressman H, et al. GFAP mutations, age at onset, and clinical subtypes in Alexander disease. Neurology. 2011;77(13):1287-94

12. Balbi P, Seri M, Ceccherini I, Uggetti C, Casale R, Fundaro C, Caroli F, Santoro L. Adult-onset Alexander disease: report on a family. J Neurol. 2008;255(1):24-30.

13. Li R, Johnson AB, Salomons G, Goldman JE, Naidu S, Quinlan R, Cree B, Ruyle SZ, Banwell B, D'Hooghe M, et al. Glial fibrillary acidic protein mutations in infantile, juvenile, and adult forms of Alexander disease. Ann Neurol. 2005;57(3):310-26.

14. Schmidt S, Wattjes MP, Gerding WM, van der Knaap M. Late onset Alexander's disease presenting as cerebellar ataxia associated with a novel mutation in the GFAP gene. J Neurol. 2011;258(5):938-40.

15. Wippold FJ 2nd, Perry A, Lennerz J. Neuropathology for the neuroradiologist: Rosenthal fibers. AJNR Am J Neuroradiol. 2006;27(5):958-61.

16. Bachetti T, Caroli F, Bocca P, Prigione I, Balbi P, Biancheri R, Filocamo M, Mariotti C, Pareyson D, Ravazzolo R, et al. Mild functional effects of a novel GFAP mutant allele identified in a familial case of adult-onset Alexander disease. Eur J Hum Genet. 2008;16(4):462-70.

17. Boczek NJ, Sigafoos AN, Zimmermann MT, Maus RL, Cousin MA, Blackburn PR, Urrutia R, Clark KJ, Patterson MC, Wick MJ, et al. Functional characterization of a GFAP variant of uncertain significance in an Alexander disease case within the setting of an individualized medicine clinic. Clin Case Rep. 2016:4(9):885-95.

18. Der Perng M, Su M, Wen SF, Li R, Gibbon T, Prescott AR, Brenner M, Quinlan RA. The Alexander disease-causing glial fibrillary acidic protein mutant, R416W, accumulates into Rosenthal fibers by a pathway that involves filament aggregation and the association of alpha B-crystallin and HSP27. Am J Hum Genet. 2006;79(2):197-213.

19. Hsiao K, Chapman P, Nilsen S, Eckman C, Harigaya Y, Younkin S, Yang F, Cole G. Correlative memory deficits, Abeta elevation, and amyloid plaques in transgenic mice. Science. 1996;274(5284):99-102.

20. Nam TS, Kim JH, Chang CH, Yoon W, Jung YS, Kang SY, Shin BA, Perng MD, Choi SY, Kim MK. Identification of a novel nonsense mutation in the rod domain of GFAP that is associated with Alexander disease. Eur J Hum Genet. 2015;23(1):72-8

21. Grunwald DJ, Eisen JS. Headwaters of the zebrafish - emergence of a new model vertebrate. Nat Rev Genet. 2002;3(9):717-24

22. Ingham PW. The power of the zebrafish for disease analysis. Hum Mol Genet. 2009;18(R1):R107-12.

23. Kawai H, Arata N, Nakayasu H. Three-dimensional distribution of astrocytes in zebrafish spinal cord. Glia. 2001;36(3):406-13.

24. Nielsen AL, Jorgensen AL. Structural and functional characterization of the zebrafish gene for glial fibrillary acidic protein, GFAP. Gene. 2003;310:123-32.
25. Bernardos RL, Raymond PA. GFAP transgenic zebrafish. Gene Expr Patterns. 2006:6(8):1007-13.

26. Lim HS, Jin S, Yun SJ. Modulation of Melanogenesis by Heme oxygenase-1 via p53 in normal human melanocytes. Chonnam Med J. 2016;52(1):45-52.

27. Kim JH, Lee SR, Li LH, Park HJ, Park JH, Lee KY, Kim MK, Shin BA, Choi SY. High cleavage efficiency of a 2A peptide derived from porcine teschovirus-1 in human cell lines, zebrafish and mice. PLoS One. 2011;6(4):e18556.

28. Urasaki A, Morvan G, Kawakami K. Functional dissection of the Tol2 transposable element identified the minimal cis-sequence and a highly repetitive sequence in the subterminal region essential for transposition. Genetics. 2006:174(2):639-49.

29. Westerfield M: THE ZEBRAFISH BOOK: a guide for the laboratory use of zebrafish (Danio rerio), 5 edn: University of Oregon Press; 2007.

30. Kimmel CB, Ballard WW, Kimmel SR, Ullmann B, Schilling TF. Stages of embryonic development of the zebrafish. Developmental dynamics : an official publication of the American Association of Anatomists. 1995;203(3):253-310

31. Delongchamp RR, Razzaghi M, Lee T. Estimating false discovery rate and false non-discovery rate using the empirical cumulative distribution function of p-values in 'omics' studies. Genes Genom. 2011;33(5):461-6.

32. Chang KE, Pratt D, Mishra BB, Edwards N, Hallett M, Ray-Chaudhury A. Type II (adult onset) Alexander disease in a paraplegic male with a rare D128N mutation in the GFAP gene. Clin Neuropathol. 2015;34(5):298-302.

33. Pareyson D, Fancellu R, Mariotti C, Romano S, Salmaggi A, Carella F, Girotti F, Gattellaro G, Carriero MR, Farina L, et al. Adult-onset Alexander disease: a series of eleven unrelated cases with review of the literature. Brain. 2008;131(Pt 9):2321-31.

34. Messing A, Head MW, Galles K, Galbreath EJ, Goldman JE, Brenner M. Fatal encephalopathy with astrocyte inclusions in GFAP transgenic mice. Am J Pathol. 1998;152(2):391-8.

35. Tang G, Yue Z, Talloczy Z, Hagemann T, Cho W, Messing A, Sulzer DL, Goldman JE. Autophagy induced by Alexander disease-mutant GFAP accumulation is regulated by p38/MAPK and mTOR signaling pathways. Hum Mol Genet. 2008;17(11):1540-55.

36. Ebrahimi-Fakhari D, Wahlster L, Hoffmann GF, Kolker S. Emerging role of autophagy in pediatric neurodegenerative and neurometabolic diseases. Pediatr Res. 2014:75(1-2):217-26.

37. Faber PW, Alter JR, MacDonald ME, Hart AC. Polyglutamine-mediated dysfunction and apoptotic death of a Caenorhabditis Elegans sensory neuron. Proc Natl Acad Sci U S A. 1999;96(1):179-84.

38. lijima K, Liu HP, Chiang AS, Hearn SA, Konsolaki M, Zhong Y. Dissecting the pathological effects of human Abeta40 and Abeta42 in drosophila: a potential model for Alzheimer's disease. Proc Natl Acad Sci U S A. 2004;101(17):6623-8.

39. Biswas P, Chavali VR, Agnello G, Stone E, Chakarova C, Duncan JL, Kannabiran C, Homsher M, Bhattacharya SS, Naeem MA, et al. A missense mutation in ASRGL1 is involved in causing autosomal recessive retinal degeneration. Hum Mol Genet. 2016;

40. Gama Sosa MA, De Gasperi R, Elder GA. Modeling human neurodegenerative diseases in transgenic systems. Hum Genet. 2012;131(4):535-63.

41. Schiffer NW, Broadley SA, Hirschberger T, Tavan P, Kretzschmar HA, Giese A, Haass C, Hartl FU, Schmid B. Identification of anti-prion compounds as efficient inhibitors of polyglutamine protein aggregation in a zebrafish model. J Biol Chem. 2007;282(12):9195-203.

42. Chen YS, Lim SC, Chen MH, Quinlan RA, Perng MD. Alexander disease causing mutations in the C-terminal domain of GFAP are deleterious both to assembly and network formation with the potential to both activate caspase 3 and decrease cell viability. Exp Cell Res. 2011;317(16):2252-66.

43. Jany PL, Hagemann TL, Messing A. GFAP expression as an indicator of disease severity in mouse models of Alexander disease. ASN Neuro. 2013;5(1):e00109.

44. Bennett RL, French KS, Resta RG, Doyle DL. Standardized human pedigree nomenclature: update and assessment of the recommendations of the National Society of genetic counselors. J Genet Couns. 2008;17(5):424-33. 